\title{
UTILIZAÇÃO DOS COMPONENTES DA CERA DAS PLANTAS, EM ESPECIAL OS n-ALCANOS, EM ESTUDOS DE NUTRIÇÃO DE RUMINANTES
}

\author{
THE UTILIZATION OF PLANT WAX COMPONENTS, ESPECIALLY \\ n-ALKANES, IN RUMINANTS NUTRITION STUDIES
}

\author{
Dimas Estrásulas de Oliveira ${ }^{1}$ Ênio Rosa Prates ${ }^{2}$
}

\section{- REVISÃO BIBLIOGRÁFICA -}

\section{RESUMO}

A utilização de métodos indiretos para estimar o consumo e a digestibilidade dos alimentos por ruminantes é bastante importante, porque pode representar uma economia de tempo, dinheiro e trabalho em relação aos experimentos convencionais e, também, por permitir tais estimativas sob condições extensivas de pastejo. Dentre esses métodos, sobressai-se o dos indicadores fecais. Várias substâncias têm sido utilizadas para esse fim. Este artigo é uma revisão de alguns aspectos sobre o uso de n-alcanos como indicadores em estudos de nutrição de ruminantes.

Palavras-chave: ceras, consumo, digestibilidade, hidrocarbonetos, indicadores, $n$-alcanos.

\section{SUMMARY}

The indirect methods used to estimate the intake and digestibility offeedstuffs by ruminants are very important because these methods represent an economy of time, money and work, in relation to conventional trials. Also, they permit estimates in grazing conditions. Among these methods, the faecal markers stand out and lots of substances have been used. This paper is a review about the use of n-alkanes as markers in ruminant nutrition studies.

Key words: digestibility, hydrocarbons, intake, markers, nalkanes, waxes.

\section{INTRODUÇÃO}

A avaliação do valor nutritivo dos alimentos fornecidos a ruminantes é um assunto de grande importância. Sua determinação envolve o conhecimento do consumo e da digestibilidade desses alimentos. A medida do consumo e da digestibilidade a campo é trabalhosa, cara e muitas vezes inviável. Por isso, a utilização dos indicadores fecais tem se sobressaído.

Dentre as várias substâncias que compõem a cera das plantas, surgiram diversas com potencial de utilização como indicadores, tais como os ácidos graxos de cadeia longa (GRACE \& BODY, 1981) e, especialmente, os hidrocarbonetos alifáticos saturados (n-alcanos) (MAYES \& LAMB, 1984).

Os n-alcanos são compostos orgânicos de cadeia aberta formados por carbono e hidrogênio, onde a diferença entre dois membros sucessivos da série é constante (são homólogos), e onde há o prefixo n (normal) para os diversos alcanos, por maiores que sejam as moléculas, desde que os átomos de carbono se encontrem em cadeia contínua, sem ramificações. Destes, os que contêm mais de 20 átomos de carbono são, também, chamados de parafinas (MORRISON \& BOYD, 1972).

\footnotetext{
${ }^{1}$ Zootecnista, MSc., Aluno do Curso de Doutorado em Ciência Animal e Pastagens da ESALQ/USP.

2 Professor, PhD., Curso de Pós-graduação em Zootecnia da Faculdade de Agronomia da UFRGS, Bolsista do CNPq, (E-mail: erprates@orion.ufrgs.br) Autor para correspondência.
} 


\section{COMPOSIÇÃO E VARIAÇÃO DAS CERAS E DO PERFIL DE n-ALCANOS DAS PLANTAS}

A presença de $n$-alcanos (parafinas) na cera de insetos e plantas já vem sendo demonstrada claramente há vários anos. CHIBNALL et al. (1934) revisaram a literatura do período entre 1926 e 1931 e encontraram citações de que várias parafinas com número de átomos de carbono entre $\mathrm{C}_{25}$ e $\mathrm{C}_{35}$ eram constituintes comuns àquelas espécies.

O interesse pela composição química das ceras das plantas aumentou com o avanço e sofisticação das técnicas analíticas como a cromatografia gasosa, a análise do espectro infravermelho e espectrometria de massas, já que os métodos mais antigos, usualmente aplicados, são demorados e pouco precisos para estudar essas complexas misturas. Os estudos quimiotaxonômicos também fizeram com que houvesse um maior interesse em investigações mais detalhadas dos constituintes da cobertura cerosa das plantas.

Após analisarem a cera superficial do azevém perene (Lolium perenne), HAMILTON \& POWER (1969) separaram-na em três frações, uma das quais constituída inteiramente de n-alcanos com comprimento da cadeia carbonada identificado como maior de $\mathrm{C}_{25}$ até $\mathrm{C}_{37}$, com maiores proporções de $\mathrm{C}_{27}, \mathrm{C}_{29}, \mathrm{C}_{31}$ e $\mathrm{C}_{33}$. As outras frações eram ésteres de alto peso molecular e álcoois, respectivamente. TULLOCH (1981) estudou a composição das ceras cuticulares de 34 espécies e em 4 subfamílias de gramíneas nativas do Canadá. Em 12 delas, os nalcanos foram os componentes em maior concentração na cera e, em 2 destas, formaram $50 \%$ da cera total, predominando o $\mathrm{C}_{29}$ e $\mathrm{C}_{31}$. O mesmo autor, em 1984, analisou a composição de 4 espécies de gramíneas Eragrostis e, em uma, os n-alcanos foram a fração maior da cera (37\% do total), identificando componentes de $\mathrm{C}_{23}$ a $\mathrm{C}_{35}$, com predominância do $\mathrm{C}_{29}$ e do $\mathrm{C}_{31}$.

Com relação ao perfil e concentração dos vários n-alcanos, KOLATTUKUDY (1976) afirmou que os hidrocarbonetos $\mathrm{C}_{29}$ e $\mathrm{C}_{31}$ são os principais alcanos do reino vegetal. TULLOCH (1976) verificou que os alcanos estão presentes em todas as ceras, podendo variar as quantidades. Comentou, ainda, que os principais alcanos contêm número ímpar de átomos de carbono, variando de $\mathrm{C}_{21}$ a $\mathrm{C}_{37}$, e que o $\mathrm{C}_{31}$ é freqüentemente o que aparece em maior concentração, seguido pelo $\mathrm{C}_{29}$. Em um estudo mais recente, OLIVEIRA et al. (1997) analisa- ram o perfil de n-alcanos de vários fenos de gramíneas, identificaram e quantificaram n-alcanos de $\mathrm{C}_{20}$ a $\mathrm{C}_{37}$, sendo mais abundantes o $\mathrm{C}_{29}$, o $_{31}$ e o $\mathrm{C}_{33}$, com predominância dos ímpares sobre os pares.

Os n-alcanos não são os únicos componentes da cera das plantas e podem até não aparecer em maior quantidade, conforme se observa na tabela 1. Depois de uma avaliação da composição da cera das folhas de cultivares de centeio (Secale cereale) e de triticale (6A190), TULLOCH \& HOFFMAN (1974) encontraram as dicetonas formando a maior parte da cera. Esses mesmos autores também encontraram essa mesma fração como maior parte em amostras de ceras cuticulares no Agropyron intermedium (1976), Elymus cinereus, Festuca ovina (1977), Andropogon halii e Andropogon scoparius (1979) e Panicum virgatum (1980). TULLOCH et al. (1980) investigaram a composição da cera cuticular de membros da tribo Triticea, 174 espécimes de 123 espécies de 22 gêneros, com resultados mostrando considerável uniformidade dentro da tribo, onde os principais constituintes da cera foram álcoois livres ou dicetonas. Álcoois livres também aparecem como principal componente das ceras cuticulares do trigo (Triticum aestivum), aveia (Avena sativa) (TULLOCH \& HOFFMAN, 1973a; 1973b), Agropyron desertorum, Bromus inermis, Elymus junceus, Phleum pratense (TULLOCH \& HOFFMAN, 1977); ácidos graxos livres, no sorgo (Sorgum vulgare) (BIANCHI et al., 1978), e hidróxi- $\beta$-dicetonas, na Poa ampla (TULLOCH, 1978).

É possível acontecer que, em determinada espécie cuja cera foi analisada, não seja encontrado um componente em maior concentração, como no caso do capim-arroz (Echinochloa crusgalli) (TULLOCH \& BERGTER, 1980). Com relação à variação da composição das ceras e do perfil de n-

Tabela 1 - Componentes comuns da cera cuticular insaponificada das plantas *.

\begin{tabular}{llc}
\hline \multicolumn{1}{c}{ Componentes } & \multicolumn{1}{c}{ Ocorrência } & $\begin{array}{c}\text { Níveis típicos nas Poacea } \\
\text { (\% da cera) }\end{array}$ \\
\hline n-Alcanos & comum, menor a maior & $3-40$ \\
Monoésteres & comum, menor a maior & $5-20$ \\
Álcoois primários & comum, menor a maior & $2-70$ \\
Ác. graxos de cadeia longa & comum, menor & usualmente $<10$ \\
Álcoois e cetonas secundários & comum, menor a maior & ------- \\
$\beta$-dicetonas & comum, menor a maior & $5-70$ \\
Hidróxi ou oxo- $\beta$-dicetonas & incomum, menor a maior & $3-50$ \\
& & \\
\hline
\end{tabular}

* Adaptado de TULLOCH (1976). 
alcanos das plantas, HORN et al. (1964) comentaram que, dependendo da idade das folhas e, possivelmente, também da parte da planta coletada, poderia haver variação nas proporções dos constituintes da cera. A esse respeito, HAMILTON \& POWER (1969) encontraram grande declínio (de 15 para 3\% do total) na quantidade de n-alcanos com menos de 25 átomos de carbono no período de crescimento de 6 a 14 dias em amostras de ceras de plantas de azevém perene (Lolium perenne). Eles concluíram que ocorre alguma variação na composição dos nalcanos durante os primeiros estádios de crescimento (6 a 8 dias) mas, após, mantêm-se num padrão constante. Recentemente, OLIVEIRA et al. (1997) verificaram uma diminuição na quantidade dos nalcanos $\mathrm{C}_{33}$ e $\mathrm{C}_{35}$ em fenos de napier (Pennisetum purpureum), conforme avançou a maturidade das plantas.

Com base na literatura, pode-se constatar algumas características sobre o perfil da fração de nalcanos da cera das plantas (temperadas e tropicais): 1) há uma predominância de n-alcanos com cadeias ímpares sobre as pares; 2) os comprimentos da cadeia de carbono detectados variam em função do tipo de estudo, mas, usualmente, estão entre $\mathrm{C}_{25}$ a $\mathrm{C}_{35}$. N-alcanos com cadeias mais curtas são encontrados, mas normalmente estão presentes em menores quantidades; 3 ) dentre os n-alcanos identificados, os encontrados em maiores concentrações são o $\mathrm{C}_{25}$, $\mathrm{C}_{31}$ e $\mathrm{C}_{33}$.

\section{USO DE COMPONENTES DAS CERAS DAS PLANTAS PARA ESTIMAR A DIGESTIBILI- DADE E O CONSUMO DOS ALIMENTOS POR RUMINANTES}

Possivelmente, há 33 anos, estava surgindo o primeiro indício de que se poderia usar os n-alcanos como indicadores fecais. ORÓ et al. (1965) verificaram a existência de uma grande similaridade entre o perfil de n-alcanos extraídos das fezes de bovinos e o da forragem consumida (Medicago arabica). Em ambos os materiais, $\mathrm{n}$ alcanos de $\mathrm{C}_{18}$ a $\mathrm{C}_{35}$ foram encontrados, predominando $\mathrm{C}_{29}, \mathrm{C}_{31}$ e $\mathrm{C}_{33}$, sendo, destes, o $\mathrm{C}_{31} \mathrm{em}$ maior quantidade. Os autores concluíram que os hidrocarbonetos com pesos moleculares relativamente altos, encontrados nas fezes de bovinos, eram oriundos da pastagem que constituía a dieta. Observaram, também, que os animais selecionaram a fração folha (quando comparada com a planta inteira) porque existiu uma melhor correlação entre o perfil de n-alcanos desta com aqueles encontrados nas fezes.
Anos após, surgiu o primeiro estudo sugerindo o uso de algum componente da cera cuticular das plantas como indicador em estudos nutricionais. GRACE \& BODY (1981) examinaram a possibilidade de os ácidos graxos de cadeia longa $\left(\mathrm{C}_{19}\right.$ a $\left.\mathrm{C}_{32}\right)$ presentes na forragem fresca servirem como substâncias indigestíveis para estimar o consumo em animais sob pastejo. Com 2 grupos de ovinos e duas dietas, as produções fecais dos ácidos graxos de $\mathrm{C}_{19}$ a $\mathrm{C}_{32}$, dentro de cada dieta, não foram significativamente diferentes das quantidades ingeridas, sugerindo que os ácidos graxos não foram absorvidos no trato digestivo.

\section{UTILIZAÇÃO DE n-ALCANOS COMO INDI- CADORES INTERNOS DE DIGESTIBILIDA- DE}

Em um estudo com ovinos que receberam uma dieta de azevém (Lolium multiflorum) e trevo branco (Trifolium repens), MAYES \& LAMB (1984) sugeriram que os n-alcanos de cadeia longa poderiam ser úteis como indicadores para determinar a digestibilidade de forragens. Os mesmos autores concluíram, também, que houve um progressivo aumento na recuperação fecal, conforme aumentou o comprimento da cadeia carbonada dos n-alcanos, sendo a recuperação do $\mathrm{C}_{35}$ de $97,5 \%$.

PIASENTIER et al. (1989) usaram amostras de alimento (Dactylis glomerata) e de fezes de ovinos, obtidas de ensaios de digestibilidade in vivo conduzidos previamente, para testar a adequabilidade dos n-alcanos com número ímpar de carbonos de $\mathrm{C}_{27}$ a $\mathrm{C}_{33}$ como indicadores de digestibilidade. A média de recuperação fecal dos n-alcanos alcançou um máximo de $83,4 \%$ com o $\mathrm{C}_{33}$, de maneira que a digestibilidade determinada foi sempre subestimada em maior ou menor extensão. Contudo, com os homólogos de maior comprimento de cadeia, $\mathrm{C}_{31} \mathrm{e}$ $\mathrm{C}_{33}$, a correlação entre os coeficientes de digestibilidade in vivo com o estimado com os referidos nalcanos foi de 0,80 e 0,84 , respectivamente.

Em um trabalho com ovelhas lactantes em pastagem de azevém perene (Lolium perenne), DOVE et al. (1990) calcularam os consumos de forragem usando estimativas de digestibilidade obtidas pelo método in vitro ou com o n-alcano $C_{35}$. Concluíram que as estimativas de consumo baseadas na digestibilidade com o $\mathrm{C}_{35}$ foram mais exatas. Segundo OHAJURUKA \& PALMQUIST (1991), as cinzas insolúveis em ácido (CIA) forneceram melhores resultados como indicador de digestibilidade, quando comparadas com o n-alcano $\mathrm{C}_{31}$. 
Recentemente, OLIVEIRA (1995) trabalhou com amostras de alimentos e fezes oriundas de experimentos convencionais de digestibilidade com ovinos realizados entre os anos de 1978 e 1982, e utilizou o n-alcano $\mathrm{C}_{35}$ como indicador interno de digestibilidade. Os coeficientes de digestibilidade obtidos com o indicador foram sempre subestimados tanto para matéria seca como para matéria orgânica, devido à recuperação média do $\mathrm{C}_{35}$ ter sido inferior a $100 \%$, mas os coeficientes de correlação obtidos entre as medidas in vivo e com indicador para matéria seca e matéria orgânica foram considerados bons ( 0,87 e 0,85 , respectivamente).

\section{UTILIZAÇÃO DE n-ALCANOS COMO INDICADORES PARA ESTIMAR O CONSU- MO DE FORRAGENS}

$\mathrm{O}$ uso dos n-alcanos presentes na cera das plantas como indicadores para estimar o consumo de forragens por ruminantes é algo relativamente recente. MAYES et al. (1986a), trabalhando com ovinos, estudaram as recuperações fecais de nalcanos $\mathrm{C}_{27}$ a $\mathrm{C}_{35}$, da forragem (Lolium perenne) e de $\mathrm{n}$-alcanos fornecidos oralmente, de cadeia par $\mathrm{C}_{28}$ e $\mathrm{C}_{32}$. As recuperações fecais dos n-alcanos presentes na forragem (de cadeia ímpar) aumentaram com o aumento do comprimento da cadeia. A do n-alcano $\mathrm{C}_{28}$ fornecido foi levemente maior que as do $\mathrm{C}_{27} \mathrm{e}$ $\mathrm{C}_{29}$ da forragem e as do n-alcano $\mathrm{C}_{32}$, fornecido oralmente, e do $\mathrm{C}_{33}$ da forragem foram as mesmas. $\mathrm{O}$ consumo médio estimado, usando o par de $\mathrm{n}$ alcanos $\mathrm{C}_{32}$ fornecido oralmente e $\mathrm{C}_{33}$ da forragem, foi idêntico ao consumo real. Os autores concluíram que estimativas exatas do consumo de forragem são possíveis de obtenção, usando simultaneamente para o cálculo um par de n-alcanos: um de cadeia par fornecido oralmente e um de cadeia ímpar da forragem.

Segundo DOVE \& MAYES (1991), a grande contribuição do trabalho citado anteriormente foi que as recuperações fecais incompletas dos nalcanos de cadeia par, fornecidos oralmente, e do ímpar da forragem não têm importância desde que sejam iguais. Assim, os erros associados com as recuperações fecais incompletas anular-se-iam no numerador e denominador da equação usada para calcular o consumo. Nota-se, então, que nas fezes somente é exigida a relação das concentrações dos $\mathrm{n}$-alcanos, natural da forragem e o sintético fornecido oralmente. Se essas forem estimadas com similares "vícios" irão anular-se. Idênticas recuperações fecais dos n-alcanos da forragem (ímpar) e do fornecido oralmente (par) são o pré-requisito para obter estimativas exatas do consumo de forragem. Assim, o método pode ser particularmente útil, permitindo estimativas não viciadas do consumo de forragem em animais recebendo suplementação alimentar.

Vários trabalhos foram encontrados na literatura científica, que confirmam a possibilidade de uso dos n-alcanos para a obtenção de estimativas do consumo de forragens. MAYES et al. (1986b) trabalharam com ovinos lactentes alojados individualmente, recebendo $1 \mathrm{~kg}$ de leite/dia, com acesso $\mathrm{ad}$ libitum à forragem fresca (Lolium perenne). Os animais foram dosados com cápsulas de gelatina contendo $\mathrm{C}_{32}$, e com base nas recuperações fecais $(81,6$ e $81,7 \%)$ usaram o par $\mathrm{C}_{33}: \mathrm{C}_{32}$ respectivamente para estimar o consumo de forragem. A discrepância média entre os consumos real e estimado foi de $0,4 \mathrm{~g} / \mathrm{dia}$. Concluiu-se que boas estimativas do consumo de forragem por cordeiros lactentes poderiam ser obtidas usando os $n$-alcanos $\mathrm{C}_{33}$ da forragem e $\mathrm{C}_{32}$ fornecido oralmente como indicadores interno e externo, respectivamente. Resultados semelhantes, trabalhando com vacas de corte, obtiveram MAYES et al. (1986c) e DILLON \& STAKELUM (1989), e com vacas leiteiras, STAKELUM \& DILLON (1990) e DILLON \& STAKELUM (1990a).

Em dois experimentos com ovinos alojados individualmente, VULICH et al. (1991) avaliaram também o uso de n-alcano sintético $\mathrm{C}_{32}$ fornecido oralmente aos animais, e $\mathrm{C}_{31}$ e $\mathrm{C}_{33}$ da forragem, para estimar o consumo. As estimativas baseadas nas relações $\mathrm{C}_{31}: \mathrm{C}_{32}$ e $\mathrm{C}_{32}: \mathrm{C}_{33}$ mostraram similares precisões no cálculo do consumo de forragem, e uma correlação de 0,92 entre o consumo real e estimado. Segundo os autores, a técnica de n-alcanos (fornecido oralmente:natural da forragem) pode dar estimativas exatas e precisas do consumo de forragem. Já CASSON et al. (1990) recomendaram que a concentração do n-alcano natural de cadeia ímpar, usado na técnica de duplo alcano (fornecido:natural), deveria ser superior a $50 \mathrm{mg} / \mathrm{kg}$ MS, embora essa concentração crítica dependa da exatidão da análise e da precisão exigida para o cálculo do consumo.

Para determinar se a técnica do duplo alcano (fornecido:natural) poderia ser usada para estimar o consumo de 7 espécies de plantas forrageiras tropicais, LAREDO et al. (1991) investigaram as concentrações do n-alcano $\mathrm{C}_{33}$ e concluíram que algumas dessas contêm insuficientes quantidades de $\mathrm{C}_{33}$ para essa medida do consumo. Alguns n-alcanos com cadeia mais curta podem ser, às vezes, usados, mas com uma redução na exatidão da estimativa nessas espécies.

DOVE $\boldsymbol{e t}$ al. (1995) utilizaram ovinos alojados individualmente, que recebiam uma dieta 
de alfafa:grãos de aveia, com três relações diferentes. O consumo do suplemento (grãos de aveia) foi estimado por um período de 6 dias, marcando os grãos com água tritiada e monitorando o acúmulo de trítio no "pool" de água corporal. Durante o mesmo período, os animais recebiam oralmente, duas vezes ao dia, cápsulas contendo os n-alcanos $\mathrm{C}_{28}$ e $\mathrm{C}_{32}$. As amostras de fezes foram obtidas diretamente do reto dos animais e os n-alcanos foram extraídos das cápsulas, das amostras de alfafa, dos grãos de aveia e das fezes. Usando três relações de pares de nalcanos: $\mathrm{C}_{28}: \mathrm{C}_{29}, \mathrm{C}_{31}: \mathrm{C}_{32}$ e $\mathrm{C}_{32}: \mathrm{C}_{33}$, obtiveram três estimativas de consumo, sendo que a obtida através do par $\mathrm{C}_{32}: \mathrm{C}_{33}$ não diferiu significativamente daquelas obtidas in vivo. Esses resultados vêm ao encontro daqueles obtidos por MAYES et $\boldsymbol{a l}$. (1986a), onde a técnica do duplo n-alcano pode ser usada para obter estimativas exatas do consumo de forragem em animais que também estão consumindo suplementos.

Para verificar a exatidão da técnica do duplo alcano na estimativa do consumo em bovinos, OLIVÁN \& OSORO (1995) utilizaram dez vacas em gaiolas metabólicas, onde recebiam feno de alfafa na proporção de $1,5 \%$ do peso vivo. Esses animais receberam, uma vez ao dia, um pélete de papel impregnado com o n-alcano $\mathrm{C}_{32}$, durante 11 dias. No mesmo período, foram coletadas amostras de fezes de cada animal. O consumo durante os últimos três dias foi estimado utilizando-se o par de n-alcanos $\mathrm{C}_{32}: \mathrm{C}_{33}$. O método subestimou o consumo com um vício médio de $11 \%$. A provável explicação encontrada pelos autores está no fato de o nível de consumo Ter sido considerado baixo $(1,5 \%$ do peso vivo) e, provavelmente, a taxa de fluxo de nutrientes através do trato digestivo foi muito lenta e, havendo um longo tempo de retenção das partículas no trato digestivo, poderia causar maior absorção do nalcano $\mathrm{C}_{33}$ do alimento, o qual estaria associado com a fase sólida da digesta. $\mathrm{O}$ mesmo não aconteceria com o $\mathrm{C}_{32}$ fornecido oralmente, associado à fase líquida, que passa mais rapidamente. Nesse caso, a recuperação fecal do $\mathrm{C}_{33}$ poderia ter sido menor do que a do $\mathrm{C}_{32}$, levando a uma subestimativa do consumo.

FRIEND et al. (1995) utilizaram o par de n-alcanos $\mathrm{C}_{31}$, natural das forragens, e $\mathrm{C}_{32}$, fornecido oralmente sob a forma de cápsula de liberação lenta, para estimar o consumo de forragens por ovinos em condições extensivas de pastejo. Usaram três linhagens da raça Merino, sob três regimes diferentes de pastejo, em duas estações do ano (inverno e verão). Coletaram amostras das forragens, observando $\mathrm{o}$ padrão de pastejo dos animais e tentando colher o mesmo que os animais estariam consumindo. Para testar se o procedimento era exato, compararam o padrão de n-alcanos, expressos como o percentual da soma dos n-alcanos de cadeia ímpar, para as amostras de forragens e fezes correspondentes. Eles encontraram alguma variação particularmente durante o verão. Salientaram, então, que, no cálculo do consumo, as amostras das forragens poderiam não ter sido verdadeiramente representativas daquelas que os animais estariam selecionando no pastejo. Concluiram que a técnica oferece um meio seguro de estimar o consumo em condições extensivas de pastejo, mas que há necessidade de se ter muito cuidado na obtenção de amostras representativas da forragem que os animais estão consumindo.

Em outro estudo para a determinação do consumo, CHAMPION et al. (1995) trabalharam com seis raças de ovinos em pastejo extensivo sobre pastagens melhoradas, em duas estações do ano, onde também utilizaram a técnica do par de nalcanos $\left(\mathrm{C}_{31}\right.$ natural $\mathrm{da}$ forragem: $\mathrm{C}_{32}$ fornecido oralmente). Utilizaram os mesmos critérios de FRIEND et al. (1995) para o fornecimento do nalcano sintético e de amostragem de forragens e fezes, concluindo que a técnica de n-alcanos se mostrou sensível o suficiente para detectar diferenças no consumo entre estações do ano, principalmente sob condições de pastagens relativamente uniformes. Neste trabalho, os autores encontraram uma melhor relação entre o padrão de n-alcanos da pastagem amostrada e o das fezes dos animais tanto no inverno como no verão.

Com o objetivo de avaliar o potencial dos n-alcanos como indicadores para predizer o consumo de vacas leiteiras submetidas a uma dieta à base de feno, UNAL et al. (1997) mantiveram três vacas da raça Holandês fistuladas no rúmen, em baias individuais, onde foram submetidas a três níveis diferentes de oferta de alimento mais $1 \mathrm{~kg}$ de cevada machacada por animal por dia. Os animais foram dosados via fístula com papel filtro impregnado com os n-alcanos $\mathrm{C}_{32}$ e $\mathrm{C}_{36}(0,5 \mathrm{~g}$ de cada). $\mathrm{O}$ consumo foi predito usando a relação do n-alcano $\mathrm{C}_{33}$ natural do alimento e os sintéticos $\mathrm{C}_{32}$ e $\mathrm{C}_{36}$ fornecidos via fístula ruminal. Segundo os autores, as melhores estimativas do consumo foram obtidas com a relação $\mathrm{C}_{33}: \mathrm{C}_{32}$, sendo que a relação $\mathrm{C}_{33}: \mathrm{C}_{36}$, consistentemente, subestimou o consumo. Sabe-se que uma das prerrogativas para que a relação n-alcano de cadeia ímpar natural do alimento e n-alcano de cadeia par fornecido possa ser usada na estimativa do consumo é que as recuperações fecais sejam similares. Neste trabalho, as recuperações fecais médias foram 0,88 para o $C_{32}, 0,93$ para o $C_{33}$ e 1,02 para o $C_{36}$, havendo, portanto, maior similaridade entre as recuperações fecais do par $\mathrm{C}_{33}: \mathrm{C}_{32}$ quando comparado com o 
par $\mathrm{C}_{33}: \mathrm{C}_{36}$.

\section{DIGESTÃO E METABOLISMO DE n-ALCA- NOS}

Conforme a literatura, as recuperações fecais incompletas de n-alcanos naturais da forragem e sintéticos fornecidos sugerem que os alcanos não são totalmente inertes no trato digestivo. Com os objetivos de determinar onde, no trato digestivo, desaparecem os n-alcanos, de verificar se há síntese de alcanos e se as perdas são devido à absorção e metabolismo, MAYES et al. (1988) trabalharam com ovinos alojados individualmente, fistulados no rúmen e com cânulas tipo "T" no duodeno e íleo terminal, para estudar a digestão e metabolismo de $n$ alcanos naturais da dieta e sintéticos fornecidos $\left(\mathrm{C}_{28}\right.$, $\mathrm{C}_{32}$ e $\mathrm{C}_{36}$ ). Eles encontraram pouco desaparecimento dos alcanos da dieta no rúmen, ceco e cólon, ocorrendo desaparecimento no intestino delgado. As perdas dos $n$-alcanos fornecidos foram mais variáveis. Eles também constataram que há síntese de nalcanos em quantidades insignificantes no trato digestivo, e que as estimativas de consumo de forragem não seriam afetadas por alcanos endógenos. $\mathrm{O}$ desaparecimento de alcanos no trato digestivo é aparentemente devido à absorção no intestino delgado, com alguma oxidação a $\mathrm{CO}_{2}$ dos alcanos absorvidos, e a microflora ruminal parece ser incapaz de sintetizar alcanos de cadeia longa.

Também sobre esse assunto, KAFILZADEH \& PARKER (1990) usaram ovinos fistulados no rúmen e com cânulas reentrantes, e compararam as recuperações de n-alcanos naturais $\left(\mathrm{C}_{31}\right.$ e $\left.\mathrm{C}_{35}\right)$ e sintéticos fornecidos $\left(\mathrm{C}_{32}\right.$ e $\left.\mathrm{C}_{36}\right)$ com as do sesquióxido de cromo em estudos de digestão intestinal. Das recuperações nos conteúdos do duodeno, íleo e fezes sugeriram que as perdas dos nalcanos ocorrem tanto no intestino delgado como no grosso.

OHAJURUKA \& PALMQUIST (1991) trabalharam com vacas da raça Holandês não lactantes, fistuladas no rúmen e com cânula duodenal tipo "T", dosadas com n-alcano sintético $\mathrm{C}_{32}$ e com sesquióxido de cromo e avaliaram o local de perda do $\mathrm{C}_{32}$. Eles concluíram que as perdas ruminais foram responsáveis pela recuperação fecal incompleta e sugeriram o uso desse n-alcano para estimar a digestão no rúmen.

\section{MÉTODOS DE FORNECIMENTO DE n-AL- CANOS SINTÉTICOS PARA ESTUDOS NU- TRICIONAIS COM ANIMAIS}

MAYES et al. (1986a) forneceram nalcanos sintéticos $\left(\mathrm{C}_{28}\right.$ e $\left.\mathrm{C}_{32}\right)$ impregnados em tiras de papel, formando péletes, e verificaram que o coeficiente de variação do conteúdo de n-alcanos dos péletes foi de 2 a $5 \%$. Propondo algo diferente,
DOVE $\boldsymbol{e t}$ al. (1989a) usaram cápsulas de gelatina com celulose em pó, nas quais a dose de n-alcanos, previamente dissolvidos em n-heptano, era pipetada sobre a celulose, e encontraram coeficiente de variação dos conteúdos das cápsulas de 1,19 a 2,6\%, indicando que o procedimento resultou em cápsulas contendo uma dose exata. DOVE et al. (1989b) compararam péletes de papel com cápsulas de gelatina e não encontraram diferenças nas recuperações fecais dos n-alcanos fornecidos aos animais.

Um outro procedimento (VULICH et al., 1991) é uma mistura de n-alcano, solvente e celulose em pó, resultando uma suspensão facilmente homogeneizada que, após evaporada e seca, é colocada em cápsulas de gelatina. A exatidão e precisão da técnica de estimar o consumo com n-alcanos fornecidos oralmente não foram afetadas pelo método de fornecimento (cápsulas de gelatina comparado com péletes de papel).

Uma das principais desvantagens do método de n-alcanos sintéticos dosados é a necessidade de fornecê-los uma ou mais vezes ao dia através de cápsulas de gelatina ou péletes de papel. Com isso em mente, DOVE et al. (1991), trabalhando com ovinos intactos e fistulados no rúmen, alojados em gaiolas metabólicas, testaram o funcionamento de um aparelho de liberação controlada de n-alcanos sintéticos $\left(\mathrm{C}_{28}\right.$ e $\left.\mathrm{C}_{32}\right)$ colocado dentro do rúmen. Com a remoção periódica do aparelho via fístula, calcularam a taxa de liberação (considerada bastante uniforme, CV de 4,07\%) e a vida útil do aparelho (25 dias). Além do aparelho de liberação controlada de n-alcanos, os animais receberam oralmente, uma vez ao dia, péletes de papel contendo o $\mathrm{C}_{36}$. As concentrações fecais dos n-alcanos foram monitoradas através da amostragem retal, e as concentrações fecais dos n-alcanos $\mathrm{C}_{28}$ e $\mathrm{C}_{32}$ do aparelho de liberação controlada alcançaram um platô 5 dias após o início da dosagem. $\mathrm{O} \mathrm{C}_{36}$, fornecido diariamente através de péletes, mostrou uma rápida elevação na concentração fecal após o início da dosagem, mas foi muito mais errática do que as do $\mathrm{C}_{28}$ e $\mathrm{C}_{32}$ do aparelho de liberação controlada.

Com as relações de concentrações fecais de um n-alcano sintético dosado e seu adjacente natural da forragem, DOVE et al. (1991) notaram que as relações $\mathrm{C}_{28}: \mathrm{C}_{29}$ e $\mathrm{C}_{32}: \mathrm{C}_{33}$ permaneceram em um nível de platô por 6 dias após a colocação do aparelho de liberação controlada, e, ao contrário, a relação $\mathrm{C}_{36}$ : $\mathrm{C}_{35}$ foi mais errática na fase de platô. Os consumos de forragem pelos animais foram calculados usando o $\mathrm{C}_{32}$ do aparelho de liberação controlada e o $\mathrm{C}_{33}$ da forragem (Lolium perenne), e o consumo médio estimado foi idêntico ao consumo real (913,3 e 913,5g/dia, respectivamente). De forma 
contrária, os consumos calculados através do $\mathrm{C}_{36}$ fornecido diariamente e do $\mathrm{C}_{35}$ natural da forragem superestimaram o consumo em 63,3g de MS/dia. Os autores concluíram que um aparelho de liberação controlada pode ser usado para o fornecimento de $n$ alcanos sintéticos em estimativas de consumo com animais em pastejo.

Recentemente, MARAIS et al. (1996) relataram outro procedimento baseado no fornecimento de n-alcanos sintéticos impregnados em partículas uniformes de uma gramínea (Pennisetum clandestinum, Hochst) suspensas em uma solução de água com goma xantana $(0,4 \%)$. Os autores mediram a massa da suspensão e das partículas da gramínea liberadas através de pistola dosadora ou seringa descartável. Conforme relataram, a pistola dosadora e a seringa liberaram quantidades exatas da suspensão, com coeficientes de variação para dez doses sendo 0,2 e $0,4 \%$, respectivamente. A massa de partículas da gramínea em suspensão foi levemente menos exata na liberação, com coeficientes de variação de 1,4 e $0,7 \%$ para dez doses, para pistola dosadora e seringa descartável, respectivamente. Os coeficientes de variação dos conteúdos de n-alcanos por dose foram $2,3 \%$ para seringa descartável e $2,6 \%$ para a pistola dosadora. Em um experimento em ovinos com fístula ruminal, os autores compararam o fornecimento de n-alcanos sintéticos $\mathrm{C}_{32}$ e $\mathrm{C}_{36}$, na forma de suspensão oral, ou colocados sob a forma de péletes de papel diretamente no rúmen, e não encontraram diferenças na relação $\mathrm{C}_{32}: \mathrm{C}_{36}$ nas fezes dos animais, quando o $\mathrm{C}_{32}$ foi dado oralmente em suspensão e o $\mathrm{C}_{36}$ colocado diretamente no rúmen via fístula, ou vice-versa. Sugeriram então que os n-alcanos sintéticos utilizados em estudos de consumo podem ser fornecidos na forma de suspensão oral.

\section{EXTRAÇÃO DE CERAS E ANÁLISE DE AL- CANOS}

O método a ser usado para a obtenção de ceras naturais depende de fatores como o local onde ocorrem, suas propriedades e, possivelmente, da contaminação com outros componentes, particularmente daqueles originados dos lipídios internos do organismo e dos do ambiente. As ceras de superfície são geralmente extraídas por algum tipo de lavagem com solvente, sendo a polaridade do solvente e a duração do tempo de lavagem escolhidos de tal maneira que se obtenha uma completa extração dos lipídios de superfície com um mínimo de extração dos internos (KOLATTUKUDY, 1976).

A esse respeito, TULLOCH (1976) comentou que a cera pode ser separada mecanicamente de poucas plantas, tais como a cana-de-açúcar, mas, para a maior parte delas, que são fontes comerciais de cera, tem sido extraída com solvente, sendo o hexano, provavelmente, o único vantajoso para a extração em grande escala, entretanto, o clorofórmio ou o cloro-metileno podem ser necessários para ceras duras, com altos pontos de fusão e para as que contêm ácidos triterpenos.

Em estudos de nutrição animal, originalmente, os alcanos eram removidos por uma extração com éter de petróleo, por 6 horas, em aparelho Soxhlet e, após, o resíduo era saponificado com uma solução alcóolica de $\mathrm{KOH}(1 \mathrm{M})$. Depois da saponificação, os extratos eram aplicados em uma coluna de sílica gel, obtendo-se no eluato final os alcanos (MAYES \& LAMB, 1984; MAYES et al., 1986a). Como esse método de extração e purificação era relativamente demorado, Mayes e Dove desenvolveram um método mais rápido (não publicado), confirmado por DILLON \& STAKELUM (1990b), que se baseia na saponificação direta das amostras, omitindo-se a extração com solvente por 6 horas em aparelho Soxhlet. Para o uso de n-alcanos como rotina em estudos de digestibilidade e consumo, essa técnica permitiria maior rapidez nas análises das amostras de alimentos e fezes.

Para a análise final das quantidades de alcanos, usa-se a técnica de cromatografia gasosa, com colunas empacotadas (MAYES et al., 1986a; PIASENTIER et al., 1989; DOVE et al., 1989a; 1989b), mas colunas capilares fornecem melhores resoluções dos picos cromatográficos sobre um amplo perfil de comprimentos de cadeia dos alcanos (KAISER, 1985; LAREDO et al., 1991; OHAJURUKA \& PALMQUIST, 1991; OLIVEIRA et al., 1997).

Com relação à preparação das amostras de alimentos e fezes, segundo a literatura, a extração de alcanos tem sido feita com amostras liofilizadas, mas seria mais simples se as amostras pudessem ser secas em estufa. DOVE \& MAYES (1991) relataram que pode haver diminuição na concentração de nalcanos com amostras secas em estufa e citam, ainda, que há dados insuficientes para dizer se isso é um fenômeno geral e que há necessidade urgente de pesquisa sobre os efeitos de diferentes temperaturas de secagem e suas interações com o tipo de amostra (diferentes forragens, forragens vs. fezes). OLIVÁN \& OSORO (1995) também encontraram diferenças nas concentrações dos n-alcanos extraídos, entre amostras liofilizadas e secas em estufa.

\section{CONCLUSÃO}


Há um grande potencial para a pesquisa utilizando-se n-alcanos como indicadores em estudos de nutrição de ruminantes, sendo necessário maior número de informações em áreas como extração, absorção, metabolismo e excreção de n-alcanos. Também há necessidade de se estabelecer uma análise de rotina de laboratório para facilitar o seu uso.

\section{REFERÊNCIAS BIBLIOGRÁFICAS}

CASSON, T., ROWE, J.B., THORN, C.W., et al. The use of natural n-alkanes in medicago and clover as indigestible markers. Proc Aust Soc Anim Prod, Armidale, v.18, p.462, 1990

CHAMPION, S.C., ROBARDS, G.E, LINDSAY, A.R., $\boldsymbol{e}$ t $\boldsymbol{a l}$. Alkane technology estimates of summer and winter herbage intake of four specialty carpet wool breeds, Romneys and Merinos. Proc of the New Zealand Society of Animal Production, Hamilton, v.55, p.130-132, 1995.

CHIBNALL, A.C., PIPER, S.H., POLLARD, A., et al. The constitution of the primary alcohols, fatty acids and paraffins present in plant and insect waxes. Biochem $\mathbf{J}$, London, v.28, p.2189-2208, 1934

BIANCHI, G., AVATO, P., BERTORTELLI, P., et al. Epicuticular waxes of two sorghum varieties. Phytochemistry, New York, v.17, p.999-1001, 1978.

DILLON, P., STAKELUM, G. Herbage and dosed alkanes as a grass measurement technique for dairy cows. Irish Journal Agric Res, Dublin, v.28, n.1, p.104, 1989

DILLON, P., STAKELUM, G. Dosed and herbage alkanes for predicting silage intake with dairy cows: The effect of concentrate type and level of feeding. In: EUR. GRAZING WORKSHOP, 7, 1990, Wagwningen. Proceedings.. Wageningen : Ed Institut voor Veevoedingsoderzoek, 1990a. Paper M5. np.

DILlON, P. STAKELUM, G. The analysis of n-alkanes in faeces and herbage. In: EUR. GRAZING WORKSHOP, 7 , 1990, Wageningen. Proceedings... Wageningen : Ed Institut voor Veevoedingsoderzoek, 1990b. Paper M8. np.

DOVE, H., FOOT, J.Z., FREER, M. Estimation of pasture intake in grazing ewes, using the alkanes of plant cuticular waxes. In: INTERNATIONAL GRASSLAND CONGRESS, 16, 1989, Nice. Proceedings... Nice : French Grassland Society, 1989a. V. II. 1660p. p.1091-1092.

DOVE, H., MAYES, R.W., FREER, M., et al. Faecal recoveries of n-alkanes of plant cuticular waxes in penned and grazing sheep. In: INTERNATIONAL GRASSLAND CONGRESS, 16, 1989, Nice. Proceedings... Nice : French Grassland Society, 1989b. V. II. 1660p. p.1093-1094.

DOVE, H., MILNE, J.A., MAYES, R.W. Comparison of herbage intakes estimated from in vitro or alkane-based digestibilities. Proceedings of the New Zealand Society of Animal Production, Hamilton, v.50, p.457-459, 1990.

DOVE, H., MAYES, R.W. The use of plant wax alkanes as marker substances in studies of the nutrition of herbivores: A
Review. Aust J Agric Res, Melbourne, v.42, p.913-952, 1991.

DOVE, H., MAYES, R.W., LAMB, C.S., et al. Evaluation of an intra-ruminal controlled-release device for estimating herbage intake using synthetic and plant cuticular wax alkanes. In: INTERN. SYMP. NUTR. HERB., 3, 1991, Penang, Malaysia. Proceedings... Penang : Malasyan Society of Animal Production, 1991. 115p. p.82.

DOVE, H., MAYES, R.W., FREER, M. Using cuticular wax alkanes to estimate herbage intake in animals fed supplements. Ann Zootech, Clermont-Ferrand, France, v.44, suppl.1, p.237, September, 1995.

FRIEND, M.A., ROBARDS, G.E., LINDSAY, A.R., et al. The relative intake of three Merino strains under different grazing regimes estimated using alkane technology. Proc of the New Zealand Society of Animal Production, Hamilton, v.55, p.127-129, 1995.

GRACE, N.D., BODY, D.R. The possible use of long chain $\left(\mathrm{C}_{19^{-}}\right.$ $\mathrm{C}_{32}$ ) fatty acids in herbage as an indigestible faecal marker. $\mathbf{J}$ Agric Sci, Cambridge, v.97, n.3, p.743-745, 1981.

HAMILTON, R.J., POWER, D.M. The chemical composition of the surface wax of Lolium perenne. Phytochemistry, New York, v.8 n.9, p.1771-1775, 1969.

HORN, D.H.S., KRANZ, Z.H., LAMBERTON, J.A. The composition of Eucalyptus and some other leaf waxes. Aust J Chem, Melbourne, v.17, p.464-476, 1964.

KAFILZADEH, F., PARKER, D.S. The use of n-alkanes as indigestible markers in studies on intestinal digestion in sheep. In: MEETING BRITISH SOCIETY ANIMAL PRODUCTION, 100, 1990, London. Proceedings... London: British Society of Animal Production, 1990. v.50. 52p. p.578-579.

KAISER, M.A. High-Resolution gas chromatography. In: GROB, R. L. (ed.). Modern practice of gas chromatography. $2^{\text {nd }}$ ed. New York : Wiley Interscience, 1985. p.159-186.

KOLATTUKUDY, P.E. Chemistry and biochemistry of natural waxes. New York: Elsevier, 1976. Introduction to natural waxes: p.1-15.

LAREDO, M.A., SIMPSON, G.D., MINSON, D.J., et al. The potential for using $n$-alkanes in tropical forages as a marker for determination of dry matter by grazing ruminants. J Agric Sci, Cambridge, v.117, n.3, p.355-361, 1991.

MARAIS, J.P., FIGENSCHOU, P.L., ESCOTT-WATSON, P.L., et al. Administration in suspension-form of n-alkane external markers for dry matter intake and diet selection studies. J Agric Sci, Cambridge, v.126, p.207-210, 1996.

MAYES, R.W. LAMB, C.S. The possible use of $n$-alkanes in herbage as indigestible faecal markers. Proc Nutr Soc, Cambridge, v.43, n.1-3, p.39, 1984. (Abstract)

MAYES, R.W., LAMB, C.S., COLGROVE, P.M. The use of dosed and herbage n-alkanes as markers for determination of herbage intake. J Agric Sci, Cambridge, v.107, n.1, p.161170, 1986a. 
MAYES, R.W., LAMB, C.S., COLGROVE, P.M. Determination of herbage intake of suckling lambs using long chain nalkanes as markers. Brit Soc Anim Prod, London, v.42, p.457, 1986b. (Abstract).

MAYES, R.W., WRIGHT, I.A., LAMB, C.S., et al. The use of long-chain n-alkanes as markers for estimating intake and digestibility of herbage in cattle. Brit Soc Anim Prod, London, v.42, p.457, 1986c. (Abstract).

MAYES, R.W., LAMB, C.S., COLGROVE, P.M. Digestion and metabolism of dosed even-chain and odd-chain n-alkanes in sheep. In: GEN. MEET. EUR. GRASSL. FED., 12, 1988, Dublin. Proceedings... Dublin : Irish Grassland Association, 1988. 527p. p.159-163.

MORRISON, R., BOYD, R.N. Química orgânica. 6 ed Boston: Fundação Calouste Gulbenkian, 1972. Cap.4: Alcanos: p.110-172.

OHAJURUKA, O.A., PALMQUIST, D.L. Evaluation of nalkanes as digesta markers in dairy cows. J Anim Sci, Champaign, v. 69, n.4, p.1726-1732, April, 1991.

OLIVEIRA, D.E. Digestibilidade determinada pelo método indireto usando n-alcanos. Porto Alegre - RS, 1995. 93p. Dissertação (Mestrado em Zootecnia) - Curso de Pósgraduação em Zootecnia, Universidade Federal do Rio Grande do Sul, 1995.

OLIVEIRA, D.E., PRATES, E.R., PERALBA, M.C.R. Identificação e quantificação de n-alcanos presentes nas ceras de plantas forrageiras. Rev Bras Zoot, Viçosa, v.26, n.5, p.881-886, Setembro/Outubro, 1997.

ORÓ, J., NOONER, D.W., WIKSTRÖN, S.A. Paraffinic hydrocarbons in pasture plants. Science, Washington, v.147, n.3659, p.870-873, February, 1965.

OLIVÁN, M., OSORO, K. The effect of drying treatment on the n-alkane analysis. Ann Zootech, Clermont-Ferrand, v.44, Suppl.1, p.238, September, 1995.

OLIVÁN, M., OSORO,K. The use of n-alkanes for estimating feed intake in beef cows. Ann Zootech, Clermond-Ferrand, v.44, Suppl.1, p.239, September, 1995.

PIASENTIER, E., PISON, S., BOVOLENTA, S. Impiego degli n-alcani megli studi sulla digeribilitá in vivo dei foraggi. Zoot Nutri Anim, Bologna, v.15, p.691-696, 1989.

STAKELUM, G., DILLON, P. Dosed and herbage alkanes as feed intake predictors with dairy cows: The effect of feeding level and frequency of perennial ryegrass. In: EUR. GRAZING WORKSHOP, 7, 1990, Wageningen. Proceedings... Wageningen : Ed Institut voor Veevoedingsoderzoek, 1990. Paper M4. np.

TULLOCH, A.P., HOFFMAN, L.L. Leaf wax of Triticum aestivum. Phytochemistry, New York, v.12, n.9, p.2217-
$2223,1973$.

TULLOCH, A.P., HOFFMAN, L.L. Leaf wax of oats. Lipids, Champaigne, v.8, n.11, p.617-622, 1973.

TULLOCH, A.P., HOFFMAN, L.L. Epicuticular waxes of Secale cereale and Triticale hexaploide leaves. Phytochemistry, New York, v.13, p.2535-2540, 1974.

TULLOCH, A.P. Chemistry of waxes of higher plants. In: Chemistry and biochemistry of natural waxes. New York: Elsevier, 1976. p.235-287.

TULLOCH, A.P., HOFFMAN, L.L. Epicuticular wax of Agropyron intermedium. Phytochemistry, New York, v.15, p.1145-1151, 1976.

TULLOCH, A.P., HOFFMAN, L.L. Composition of epicuticular waxes of some grasses. Can J Bot, Ottawa, v.55, n.8, p.853857, 1977.

TULLOCH, A.P. Epicuticular wax of Poa ampla leaves. Phytochemistry, New York, v.17, p.1613-1615, 1978.

TULLOCH, A.P., HOFFMAN, L.L. Epicuticular waxes of Andropogon halii and A. scoparius. Phytochemistry, New York, v.18, p.267-271, 1979.

TULLOCH, A.P., HOFFMAN, L.L. Epicuticular wax of Panicum virgatum. Phytochemistry, New York, v.19, p.837839, 1980.

TULLOCH, A.P., BAUM, B.R., HOFFMAN, L.L. A survey of epicuticular waxes among genera of Triticea. 2. Chemistry. Can J Bot, Ottawa, v.58, p.2602-2615, 1980.

TULLOCH, A.P., BERGTER, L. Epicuticular wax composition of Echinocloa crusgalli. Phytochemistry, New York, v.19, p.145-146, 1980.

TULLOCH, A.P. Composition of epicuticular waxes from 28 genera of Graminea: Differences between subfamilies. Can J Bot, Ottawa, v.59, p.1213-1221, 1981.

TULLOCH, A.P. Epicuticular waxes of four Eragrostoid grasses. Phytochemistry, New York, v.23, n.8, p.1619-1623, 1984.

UNAL, Y., GARNSWORTHY, P.C., GORTON, P. The use of nalkanes for prediction of intake in dairy cows. In: ANNUAL MEETING OF THE BRITISH SOCIETY OF ANIMAL SCIENCE - SCARBOUROUGH, 1997, Penicuick, Proceedings... Scarbourough : British Society of Animal production, 1997. 208p. p.137.

VULICH, S.A., O'RIORDAN, E.G., HANRAHAN, J.P. Use of $n$-alkanes for estimation of herbage intake in sheep: accuracy and precision of the estimates. J Agric Sci, Cambridge, v.116, n.2, p.319-323, 1991.

Ciência Rural, v. 30, n. 3, 2000. 